Rev. Chil. Pediatr. 67 (6); 267-271, 1996

\title{
Registro continuo por 24 horas de pH esofágico en niños
}

\author{
Carlos Manterola D.'; Jacqueline Fierro J. ${ }^{2}$; Eduardo Hebel W. 2; Galicia Montecinos L. ${ }^{3}$ \\ Continuous ( $24 \mathrm{~h}$ ) intraesophageal $\mathrm{pH}$ monitoring \\ in children with gastroesophageal reflux
}

\begin{abstract}
Introesophageal pH changes were andlyzed through 24 hour esophageal pH monitoring in 24 pedialric 114 bemale) patients with clinically, radiologically or endoscopically proven gastroesophageal reflux. whose oge averaged 5.8 years |range 1 to 14 years\}. Dala were recorded by means of a Digitropper Mork II Gold pH recorder and anolized by Boix-Ochoo or DeMeester methods cepending on patients age. Resulis were os foliows (mean values it atherwise not indicaled': total number of eflux episodes by 24 hours: 94; number of reflux episodes longer than 5 minutes: 2: longest reflux duration: 8 minutes; lotal lower esophageol time under $\mathrm{pH} 4.0$ or lower: 05 minutes; percentage of overall time under reflux $5.4 \%$. The besi relaled symplom to gastroesophageal reflux episodes was pyrosis $(69 \%)$.
\end{abstract}

(Koy words: esophageal ph, moni:oring, gastroesophageal reflux, esofagitis, esophagus.|

La medición continuada de $\mathrm{pH}$ esofágico en el tercio distal del esofago durante un tiempo prolongado permite registrar sus variaciones cada vez que se introduce jugo gástrico en el esófago y constituye un excelente recurso en el diagnóstico de la enfermedad por reflujo gastroesofágico (ERGE) $)^{1-3}$. Al comicnzo era un procedimiento reservado solo para investigación clínica, pero los sistemas de registro portátiles y el análisis computadorizado de los datos ${ }^{4}$ han permito su aplicación en la evaluación clínica del reflujo gastroesofágico (RGE), identificando su presencia, determinando su magnitud, y estimando la capacidad del esófago para eliminar el líquido refluido. Se considera la mejor prueba diagnóstica en pacientes con ERGE, debido a su elevada sensibilidad y especificidad ${ }^{3-8}$. En esta comunicación se describe la experiencia inicial en el registro continuo de $\mathrm{pH}$ intraeso-

1. Unidad de Motilidad Digestiva, Servicio y Departamento de Cirugía. Hospital Regional de Temuco y Universidad de La Frontera, Temuco.

2. Unidad de Gastroeoterología, Servicio y Departamento de Pediatría. Hospital Regional de Temuco y Universidad de La Fronlera. Temuco.

3. Servicio de Cirugía Infontil y Departamento de Pediatría. Hospital Regional de Temuco y Universidad de La Frontera, Temuco. fágico de 24 horas en pacientes pediátricos de un hospital regional.

\section{Material y Método}

La Unidad de Motilidad Digestiva del Departamento y Servicio de Cirugía de la Universidad de La Frontera y Hospital Regional de Temuco, comenzo a funcionar corno tal en 1993, realizando manometría esofágica". medición ambulatoria intraesofágica e intragástrica de $\mathrm{pH}^{\mathrm{B}, 11,11}$ y manometría del esfínter de Oddi ${ }^{12,13}$ para pacientes adultos. Más tarde, en los últimos 12 meses, se incorporó en forma paulatina el registro continuo de $\mathrm{pH}$ intraesofágico en individuos menores de 15 años $^{14}$

La serie cuyos resultados se exponen, estấ conformada por 10 hombres y 14 mujeres de uno a 14 años de edad (media 5.8 años). La hipótesis de diagnóstico se fundarnentó en todos en síntomas (regurgitación, tos nocturna, disfagia o pirosis), hallazgos radiologicos (reflujo espontáneo o tras prueba de sifonaje) o signos endoscópicos de esofagitis. Según la clasificación de Savary' 15 en 10 pacientes (4].7\%) la endoscopía fue norma]; en otros 10 casos $(41,7 \%)$ se cncontró esofagitis, de grados I o II; en 3 niños había esofagitis grado III $(12,5 \%)$; y en uno $(4 . J \%)$ estenosis esofágica, que había sido Iratada con dilataciones. No se describió metaplasia de Barret.

Las determinaciones se realizaron con un medidor de pH Digitrapper Mark II Gold dotado de una sonda de antimonio monocristalino modelo 1010 y electrodo de referencia (Syneclics Medical, Estocolmo). El instrumento se culibra antes de cada procedimiento, introduciendo los electrodos de referencia y registrando $\mathrm{pH}$ en 
soluciones de calibración tamponadas durante 3 minutos, primeró en solución tampón pH 7.0 y luego a $\mathrm{pH}$ 1,0 s.to. Entretanto se lavan con agua deslilada y secan ambos electrodos. A los pacientes en ayunas (en la mayoría de lo casos previamente hospitalizados) se introduce el electrodo de $\mathrm{pH}$ por vía nasal, siteando $\$ u$ extremo distal $5 \mathrm{~cm}$ por encima del borde superior del esfinter csofágico inferior (EEI). lo que se comprueba por el cambio de $\mathrm{pH}$ ácido a alcalino mediante retirada del electrodo desde el estómago, radioscopía forácica y radiografía de tórax en proyección posteroanterior. EI electrodo de referencia sc pone en la pared anterior del tórax. Los pacientes pexmanecen en bipedestación o en decúbito supino a libre demanda. Durante la prueba no se restringió el tipo de conida, bebida o hábito de vida, pero no se administraron medicamentos que pudiesen modificar o altetar el resultado del examen (antiácidos, drogas procinéticas, bloqueadores $\mathrm{H} 2$, de la bomba de protones, etc.), los que fueron suspendidos 72 horas antes. Se instruyó a los padres o enfermera a cargo de la sala para que trascribiesen el horario de comidas y cambios de posición de los pacientes, así como la aparición de sintomas que motivaron la primera consulta en una hoja de datos para este proposito $4,5 . \gamma_{+} 10$.

Se registró número total de episodios de reflujo (NTER), número de reflujos mayores de 5 ininutos, episodio más largo de reflujo, tiempo de exposición de la Inucosa al acido o tiempo total del reflujo (TTR), porcentaje de tiempo total de reflujo (\% TTR), puntaje final según DeMeester y el inicio de síntomas. Este úlıímo con la finalidad de poder determinar la relación de éstos con episodios de reflujo, y reconocer de esta forma las molestias derivadas de la enfermedad de aquéllas relacionadas con otras entidades coexistentes ${ }^{8,11,18}$.

El analisis de las variables fue desarollado inediante un sistema matemático de valoración propuesto por BoixOchoa, o por el de DeMeester ${ }^{A .17}$, según la edad del paciente (DeMeester sobre los 10 años). Esto lue procesado mediante un programa computacional Esophagogram 5.7 de Synectics Medical, Estocolino. Todas clías fueron analizadas en bidepedestación, decúbito supino y periodos prandial $y$ postprandial. S6́lo se efectuó análisis para episodios de reflujo ácido. Los datos de todos los parámetros estudiados se expresan $\bullet$ medias \pm desviación estándar (DE).

\section{Resultados}

El estudio fue bien tolerado en todos los casos. Sólo en dos pacientes $(8,3 \%)$ fue completamente normal, en los 22 restantes $(91,7 \%)$ se registraron alteraciones de alguna de las variables analizadas.

En la tabla I se describe la media, mínimo y máximo registrados para las variables analizadas: el puntaje final (DeMeester) fue realizado s6́lo en 19 pacientes mayores de 10 años de edad.

El tiempo total de reflujo fue inferior a $3,6 \%$ (considerado dentro de la categoría aceptable) en 10 casos $(41,7 \%)$; y superior a $3,6 \%$ en los 14 pacientes restantes $(58,3 \%)$.

De los sujetos con TTR $<3,6(\mathbf{n}: 10)$, sólo dos pacientes tuvieron menos de 40 episodios de reflujo en 24 horas de registro; del resto, tres casos tuvieron 41 y 50 episodios; otros tres entre 51 y 100 episodios; los dos restantes mostraron sobre 101 episodios de reflujo al día; sólo cn uno de cstos pacientes los episodios de reflujo fueron de 5 o más minutos de duración, siendo el más largo de 16 minutos; en siete pacientes se registró reflujo en decúbito supino, en de ellos más de 35 episodios. Un paciente fue el que presentó, además, tres episodios de duración superior a los cinco mi-

Tabla 1

Medición de pH esofágico: resultados en 22 pacientes con registros anormales

\begin{tabular}{|c|c|c|c|}
\hline Variable & Media $\pm D E$ & Mínimo & Máximo \\
\hline Total episodios reflujo & $94 \pm 73$ & 6 & 292 \\
\hline Episodios reflujo en supino & $43 \pm 56$ & 0 & 195 \\
\hline Episodios reflujo postprandiales & $53 \pm 58$ & 0 & 197 \\
\hline Episodios $>5$ minutos & $2 \pm 2$ & 0 & 8 \\
\hline Episodios $>$ de 5 min en supino & $1 \pm \mathbf{I}$ & 0 & 8 \\
\hline Episodio más largo (min) & $8 \pm 5$ & I & 16 \\
\hline Tiempo total de $\mathrm{pH}<4,0$ (min) & $65 \pm 51$ & 3 & 174 \\
\hline Tiempo de $\mathrm{pH}<4,0$ en supino (min) & $27 \pm 44$ & 0 & 174 \\
\hline \% total tienipo de reflujo & $5.4 \pm 4.9$ & 0,3 & 21,4 \\
\hline Puntaje final de DeMeester & $19.8 \pm 11,2 *$ & 1,2 & 44,9 \\
\hline
\end{tabular}

*: el n en este caso es de 19 (pacientes de edad superior a 10 años) 
nutos en estas condiciones. El TTR fue inferior a $\operatorname{los} 30$ minutos en 24 horas en 8 casos, sin embargo, en 2 pacientes superó los $45 \mathrm{mi}$ nutos en 24 horas, alcanzando el mayor los 52 minutos. DeMeester considera como normal un puntaje final de 14,7 puntos: dos de los 8 pacientes en que se calculó dicho baremo en este subgrupo presentaron puntuaciones superior a ésta. La calificación final fluctuó entre 1,2 y 18,2 puntos, 10 que a simple vista, y asociado a \% TTR inferior a 3,6, signiticaría un examen normal, a pesar de lo cual sólo dos de estos pacientes mostraron ausencia de alteraciones en los otros criterios estudiados.

Entre los 14 niños con tiempo total de reflujo $>3,6$ (n:4), sólo tres tuvieron menos de 50 episodios en 24 horas, cuatro presentaron entre 51 y 100 episodios; cinco entre 101 y 200 episodios; y en dos se registraron entre 201 y 300 episodios de reflujo al día. Solamente en un paciente no se registraron reflujos de más de 5 min de duración. La media de la duración en los pacientes restantes fue $10 \mathrm{~min}$, máximo $16 \mathrm{~min}$. En todos $l o s$ casos de este subgrupo se registraron episodios de reflujo en decúbito supino (media 61 episodios de reflujo nocturno o en decúbito supino): en ocho los había mayores de $5 \mathrm{~min}$ de duración, que en dos ocurrieron más de 5 veces. El TTR fue mayor que $30 \mathrm{~min}$ en todos los casos de cste subgrupo y el promedio fue de 96 minutos en 24 horas (margenes 34 a 174 minutos). En 1 I de los 14 pacientes de este subgrupo se pudo calcular el puntaje final de DeMeester, que en dos fue inferior al límite máximo normal. La calificación final fluctuó entre 14,2 y 44,9 puntos, lo que hace necesario insistir en que e] informe final de un registro continuo de $\mathrm{pH}$ de 24 horas debe incluir todos los critcrios de significación, ya que la sola consideración al tiempo total, su porcentaje o la calificación final, en forma aislada pueden conducir a crror.

S6lo en 11 pacientes (46\%) se registraron síntomas durante el desarrollo del examen (se trataba de niños mayores de 5 años, en los que se pudo obtener información acerca de la aparición de los síntomas en el curso del procedimiento). Estos fueron pirosis, regurgitación, vómitos, disfagia, tos, ahogos y dolor torácico. Los más significativos fueron pirosis $(69 \%)$, regurgitación $(42 \%)$, disfagia $(4 I \%)$ y tos $(20 \%)$. Si bien los pacientes manifestaron varios sínto- mas durante el registro, muchos no guardaban relación con los episodios de reflujo, lo que tal vez explique su gran dispersión. Sólo 5 de 10 pacientes con \% TTR menor a $3,6 \%$ fueron sintomáticos; entre los cuales tuvieron relaciones más significativas con los episodios de reflujo la disfagia $(67 \%)$ y la tos $(25 \%)$. S6lo 6 de 14 pacientes con \% TTR mayor a $3,6 \%$ tuyieron síntomas, siendo regurgitación $(71 \%)$ y pirosis $(69 \%)$ los que, en este caso, se relacionaron más significativamente con reflujo.

\section{Comentario}

El registro continuo y prolongado de $\mathrm{pH}$ intraesofágico se ha conștituido en el "patrón oro" para el diagnóstico y seguimiento de pacientes adultos y niños con ERGE. Sus indicaciones se han ido estableciendo con el tiempo 4,8,17,19-24 y en la actualidad son bastante precisas (tabla 2). Si bien el registro de $\mathrm{pH}$ intraesofágico puede no ser considerado imprescindible cuando el diagnóstico de reflujo se ha constatado por medios clínicos u otras pruebas (radiología o endoscopía), lo es cuando - con estos antecedentes- se decide someter al paciente a cirugía, eventualidad en que idealmente debe rcalizarse el estudio en el preoperatorio y postoperatorio, a modo de certificación de calidad de la cirugía antirreflujo.

La medición ambulatoria de $\mathrm{pH}$ esofágico ha reemplazado paulatinamentc al registro fijo, gracias a los progresos de la electrónica y la informática, que permiten al paciente desarrollar sus actividades cotidianas sin más limitación que la estética e interpretar el examen en forma rápida y exacta, si bien el costo total de estos sistemas es alto ${ }^{25}$. Para proteger los equipos y en pro de la confiabilidad de los resultados nuestra práctica es hospitalizar al paciente. La prueba de 24 h parece ser más confiable que las más breves de $3,8,12$ y 18 horas ${ }^{1,26}$.

Para interpretar los resultados del examen hemos adoptado las variables propuestas por Johnson y DeMeester en adultos ${ }^{4,27}$, y las de Boix-Ochoa en pediatrif ${ }^{17}$, contando en el trazado de 24 horas el número de episodios de reflujo, el porcentaje del tiempo total de que corresponde a reflujo, en decúbito supino y en ortostatismo, en el que el pH intraesofágico es inferior a 4,0, el número de reflujos de dura- 
Tabla 2

Indicaciones para medir $\mathrm{pH}$ esofágico en 24 horas

\begin{abstract}
Detección de RGE oculto, o demostración de re]ación con síntomas
Pacientes con síntomas atípicos de RGE (dolor torácico, abdominal, síntomas respiratorios, irritabilidad infantil que persisten a pesar de tratamiento medicamentoso)

Síntomas laringeos (tos, estridor laringeo, laringitis posterior)

Neumonía recurrente

Paciente con síntomas de RGE sin respuesta al tratamiento medico o en los que no se ha podido objetivar la existencia de reflujo por otros métodos

Evaluación del tratamiento de pacientes con RGE

Paciente con RGE que requiere de tratamiento quirórgico

Pacienle con recidiva de los sintomas de RGE tras cirugía antirreflujo

Pacienle con dario neurologico grave, en quien se indica alimentación a través de gastrostonía
\end{abstract}

RGE; reftujo gastroesofágico

ción superior a 5 minutos y la duración en minutos del reflujo más largo $0^{1,3-5,8}$. Los valores normales para estos índices han sido obtenidos en sujetos asintomáticos tanto en población adulta ${ }^{28}$ como pediátrica ${ }^{29,30}$. Dado que se conoce la sensibilidad y especificidad de cada una de las variables en el diagnóstico de la $\mathrm{RGE}^{31.32}$, se han hecho varios intentos de reunir todos los datos en uno solo mediante un sistema matemático de valoración. El que ha logrado mayor difusión es el propuesto por DeMeester ${ }^{4}$, aunque se sigue considerando el tiempo de exposición de la mucosa esofágica al ácido como el parámetro con mayor poder discriminatorio 25,33 . No se puede completar un informe de mediciones de $\mathrm{pH}$ esofágico sin correlacionar los síntomas anotados por el paciente durante la exploración con las variaciones del $\mathrm{pH}$ abservadas en el trazado $20,21,34,35$. Si bien parece haber mejor correlación entre sintomas y episodios de reflujo en pacientes con tiempo total de reflujo superior a $3,6 \%$, el pequeño número de casos de la serie descrita no permite obtener conclusiones con apoyo estadístico por el momento.

Se insiste en la necesidad de analizar en forma concienzuda cada una de las variables antes mencionadas, debido a que la normalidad de sólo uno o dos de ellos no implica que el estudio sea realmente negativo para RGE.

\section{Resumen}

Se describen los resultados del registro continuo, por $24 \mathrm{~h}$, de pH esofágico en 24 pacientes con reflujo gastroesofágico, 14 de ellos mujeres, de 5,8 años (l a 14) años de edad cuyo diagnóstico se sustentaba en bases clínicas, radiológicas o endoscópicas. Se empleó un sistema de registro Digitrapper Mark II Gold y un sistema de valoración de las variables BoixOchoa o de DeMeester, según la edad del paciente. Se registraron el número total de reflujos, los de más de 5 min de duración, el episodio más largo de reflujo, el tiempo de exposición de la mucosa al ácido (TTR), el porcentaje de tiempo total de reflujo, se calculó el puntaje final según DeMeester y el índice de síntomas en relación al reflujo. El estudio fue bien tolerado en todos los casos. Sólo en dos pacientes $(8,3 \%)$ los resultados fucron normales, en los 22 restantes $(91,7 \%)$ se registraron alteraciones en alguno de los criterios analizados. Las medias de los registros obtenidos fueron: número total de reflujos 94; número de episodios de reflujos mayores de 5 minutos 2; episodio de reflujo más largo $8 \mathrm{~min}$; tiempo total de $\mathrm{pH}$ inferior a 4 fue 65 minutos y porcentaje del tiempo lotal de reflujo $5,4 \%$.

(Palabras clave: reflujo gastroesofágico, esofagitis, esófago, $\mathrm{pH}$ esofágico.) 


\section{Referencias}

1. Grunde L, Kuster E. Toledo-Pimentel V, Ros E: La pHmetria tsofágica. Medicina Integral 1992; 20: 38-45.

2. Spencer J: Prolonged pH recording in the study of gastroesophageal reflux. Br J Surg 1969; 56; 912-914.

3. Johnson LF. DeMeesier TR: Twenty-four-hour $\mathrm{pH}$ monitoring of the distal esophage: a quantitative measure of gastroesophageal reflux. Am J Gastrocnterol 1974; 62: 325-332.

4. DeMeester TR, Wang CL. Wemly JA: Technique, indicatons and clinical use of 24 hours $\mathrm{pH}$ monitoting. J Thorac Cardiovasc Surg 1980; 79: 656-670.

5. Manterola C: pHonetría esofágica. Revista Médica del Sur 1992; 17: 45-48.

6. Fich $K H$, DeMeester $T R$. Albertucci $M$ : Specifitity and sensivity of objective diagnosis of gastroesophageal reflux disease. Surgery 1987; 102: 575-580.

7. Murpity D, Yuan Y, Casiell DO: Does the intraesophageal reflux disease. Strgery 1987; 102: 575-580.

8. Manternla $C$. Lojpez A, Yúfez $P$, et al: Análisis de pHmetría intracsofágica en 40 pacientes con reflujo gastroesofágico. Revista Chilena de Cirugía 1995; 47: 223-229

9. Manterola C. Hofmann E, Gutiériez R. ef al: Manometría esofágica. Puesta en marcha dé una técnica exploratoria en el Hospiral Regional de Tenuco. Libro de Resúmenes de las XV Jornadas de la Sociedad Mêdica de Cautín. Temuco 1993.

10. Manterola $C$, Gutiérrez R. Barroso MS, Yañez P: Estudio de parámetros molores esofágicos en 20 voluntarios sanos. Cuadernos de Cirugla de la Universidad Austral de Chile 1995: 9: 14-19.

11. Manserola $C$, López A. Zúñiga J, el al pHmetría esfágica en pacientes con RGE. Gastroenterología La+ tinoamericana 1994; 5 (Supl.4).

12. Manterola $C$. De Aretrabala X: Resultados de la manometria endoscópica del esfinter de Oddi. Gastroenterología Latinoamericana 1994: 5 (Supl.4).

13. Manrerula C, De Aretrabata X: Manometría endoscópica biliar $y$ del esfínter de Oddi. Descripción de Ia técnica y primeros tesultados. Revista Chilena de Cirugía 1995; $47: 558-562$.

14. Manterola C. Fierro J. Hehel E. Monsecinor $G$ : Registro continuo de pH intraesofágico en niños. Implementación de la técnica y primeros resultados en el Hospital Regional de Temuco. Libro de Resúmenes del XXIIt Congreso Nacional de Cirigía Pediátrica. Trailanqui 1995.

15. Savary $M$, Miller $G$ : The esophagus: Handbook and Atlas of Endoscopy. Gassinan, Solothum, 1978.

16. Hampton FJ, MacFatyen UM. Mayberyy JF: Variations in results of simultaneous ambulatory esophageal pH monitoring. Dig Dis Sci 1992: 37: 506-512.

17. Boix-Ochoa J, La Fuente JM, Gil-Verner $J N$ : Twenty-four hour esophageal $\mathrm{pH}$ monitoring in gastroesophageal reflux. J Pediatr Surg 1980; 15: 74-78.

18. Csendes A, Henriquez A. Quezada $S$ : Estudio continuo del $\mathrm{pH}$ intraesofágico durante 24 horas. Revista. Chilena de Cirugíz 1993; 45: 94-96.
19. Colleti RB. Christie $D L$, Orenstein SR: Indications for pediatric esophageal $\mathrm{pH}$ monitoring NASPGN Project Team Drafı 1994: 3: 1-16.

20. Breumelhof $R$, Sncut AJ: The sympton sensitivity index: a clinically important parameter in 24-hour esophageal $\mathrm{pH}$ recording. Am J Gastroenterol 1991; 86: 160-164

21. Johnston BT, McFarland RJ. Collins JS, et al: Symptom index as a marker of gasiroesophageal reJux disease. Br J Surg 1992; 79: 1054-1055.

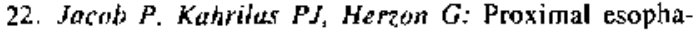
geat pH-metry in patients with reflox laryngitis. Gastroenterology 1991; 100: 305-310.

23. Contencin P, Nancy P: Gastropharyngeal reflux in infants and children. Arch Otolaryngol Head Neck Surg 1992: 118: 1028-1030.

24. Orensiein $S R$ : Controversies in pediatric gastroesophageal reflux. J Pediatr Gastroenterol Nutr 1992; 14: $338-348$.

25. Grande L, Kuster E, Toledo-Pimentel V, Ros E. La pHmetría esofágica. Medicina Integral (España) 1992: $20: 38-45$

26. Emde C. Gamer A, Blum AL: Technical aspects of intraluninal ph-metry in man: curreds status and recomendations, Gut 1987; 28: 1177-1 188.

27. Johnson LF, DeMeester TR: Twenty-four hour $\mathrm{pH}$ monitoring of the distal esophagus: a quantitative measure of gastroesophageal reflux. Am J Gastroenterol 1974; 62:325-332

28. Tytgat $G N$, Benner $J R$. Dent J, Joelssom B: Esophageal $\mathrm{pH}$ moniloring-normal and abnormal. Gastroenterul Intern 1989; 3: 14I-149.

29. Vandenplar Y, Sacré-Smits L: Continuos 24 hour esophages pH monitoring in 285 asymptomatic infants 0-I5 months old. J Pediatr Gastroenterol Nutr 1987; 6: 2201-224.

30. Vandenplas $\gamma$, Goyvatrts $R N$. Helven $R$, Sacré-Smiss $L$ : Gastroesophageal reflux, as measured by 24 -hour $\mathrm{pH}$ monitoring in 509 healthy infant dealh syndrome. Pediatrics 1991: 88: 834-840.

31. Fuch $K h$, Deblester $T R$, Aberneci $M$ : Specificity and sensitivity of objective diagnosis of gastroesophageal reflux disease. Surgery 1987; 102: $575-580$.

32. Pajot A, Grande L, Ros E. Pera C: Utility of inpatient 24-howr intraesphageal $\mathrm{pH}$ monitoring in diag. nosis of gatroesophageal reflux. Dig Dis Sci 1988; 33: $1134-1140$.

33. Marphy $D, Y_{\text {luan }} Y$. Castell DO: Does the intraesophageal pH probe accurately detect acid reflux?. Dig Dis Sci 1989; 34: 649-656.

34. Word $B W$, Wt WC, Richter JE. et al: Ambulatory 224-hours esophageal $\mathrm{pH}$ monitoring technology Searching for clinical application. J Clin Gastroenterol 1986; 8 (Supl. I): 59-67.

35. Weiner GJ, Richter $J E$, Cooper $A$, et al: The sympton index: A clinical importat parameter of ambulatory 24-hour esophageal pH monitorin. Am J Gastroenterol 1988; 83: 385-361. 